\title{
Effects of syntrophic interactions on methyl-mercury generation
}

\author{
DWAYNE ELIAS ${ }^{1}$, REGINA WILPISZESKI ${ }^{2}$, ANN \\ WYMORE $^{1}$, CAITLIN GIONFRIDDO ${ }^{1}$ AND MIRCEA \\ PODAR $^{1}$
}

\author{
${ }^{1}$ Oak Ridge National Laboratory \\ ${ }^{2}$ Oak Ridge National Lab \\ Presenting Author: eliasda@ornl.gov
}

Methylmercury (MeHg) contamination in aquatic ecosystems remains a major public health concern. The $h_{c c A B}$ genes that methylate $\mathrm{Hg}$ are widespread in anaerobic environments, but gene abundance does not predict $\mathrm{MeHg}$ concentration and the factors that control $\mathrm{MeHg}$ generation remain unclear. Syntrophic interactions between microbial species have been suggested as one factor that can enhance $\mathrm{MeHg}$ production under some conditions. Both community member abundance and gene expression profiles can influence $\mathrm{Hg}$-methylation rates, kinetics, and extents, but these factors have primarily been measured in pure cultures or in situ communities. The details governing those interactions are not well characterized. We have used a systems biology approach to understand the effects of syntrophic interactions on $\mathrm{Hg}$ methylation using microbial pairings informed by the methylating population of the Oak Ridge National Lab East Fork Poplar Creek (EFPC) study site, a natural stream that has been contaminated with $\mathrm{Hg}$ from upstream sources. Closely related methylating and non-methylating species were characterized across a complete growth curve in defined media to establish baseline levels of cellular metabolism, growth rate, $h g c A B$ expression levels, and $\mathrm{MeHg}$ production during batch growth. Species were combined under the same geochemical conditions so as to build on earlier results to determine whether the effect on Hg-methylation might be additive, subtractive or exponentially increased by complex multi-species interactions. Any observed physiological changes between single-species and co-cultures should reflect the presence of the other organism(s). Precise measurements of cell counts, $\mathrm{Hg}$ methylation rates, electron donors and acceptors, organic acids, sulfide measurements, and expression levels provided a view of the phenotypic effects of multi-species interactions on $\mathrm{Hg}$-methylation that would be difficult or impossible to extract from single cultures or uncontrolled natural communities. 